\title{
Contributing Factor of Proximal Arch Dilation in Patients with Bicuspid Aortic Valve-Wall Shear Stress or Upward Extension of Ascending Aorta Dilation?
}

\author{
Fei Li, MD,${ }^{1,3}$ Qi Gao, PhD, ${ }^{2}$ En Qiao, MD,${ }^{1}$ Gang Yin, BS, ${ }^{3}$ Ru-Jiao Zhang, MD,${ }^{4}$ Shi-Hua Zhao, MD, ${ }^{3}$ \\ Wei Wang, $\mathrm{MD}^{1}$

\begin{abstract}
${ }^{1}$ Department of Structural Heart Disease, Fuwai Hospital, Chinese Academy of Medical Sciences and Peking Union Medical College, Beijing, China; ${ }^{2}$ Institute of Fluid Engineering School of Aeronautics and Astronautics, Zhejiang University, Hangzhou, China; ${ }^{3}$ Department of Magnetic Resonance Imaging, Fuwai Hospital, Chinese Academy of Medical Sciences and Peking Union Medical College, Beijing, China; ${ }^{4}$ HeBei University Health Science Center; Baoding, China
\end{abstract}

\section{ABSTRACT}

Purpose: The dilation of proximal arch (PArc) was suspected to develop as a result of valve-related hemodynamics or in consequence of the upward extension of the ascending aorta (AAo) dilation. We aimed to investigate the one that might be the possible contributing factor in patients with bicuspid aortic valve (BAV).

Methods: All enrolled BAV patients underwent fourdimensional flow magnetic resonance imaging. Contouraveraged circumferential wall shear stress (WSScirc,avg) and the diameter of the middle of AAo (mid-AAo) were compared between the patients with and without PArc dilation. Additionally, we analyzed the correlation between WSScirc,avg and aortic diameter at PArc section, as well as the correlation between the diameter of mid-AAo and that of PArc.

Results: No significant difference was observed in WSScirc,avg at PArc section between the patients with and without PArc dilation $(P=.621)$. However, the diameter of mid-AAo in the patients with PArc dilation was higher than those without it $(P=.007)$. In addition, the aortic diameter did not correlate with the WSScirc,avg at PArc level $(\mathrm{R}=$ $-0.068, P=.701)$. The correlation was observed between PArc diameter and mid-AAo diameter $(\mathrm{R}=0.521, P=.002)$.

Conclusion: Hemodynamics may not contribute to the development of PArc dilation. PArc diameter correlated with mid-AAo diameter, implying PArc dilation may be secondary to the upward extension of AAo dilation. The influence of AAo dilation extending upward could be treated by AAo replacement; hence, a selective approach to transverse PArc replacement might be appropriate.

\section{INTRODUCTION}

Bicuspid aortic valve (BAV) is one of the most common congenital heart diseases appearing in $1 \%$ to $2 \%$ of the general

Received February 12, 2020; received in revised form April 30, 2020; accepted April 30, 2020.

Correspondence: Shi-Hua Zhao and Wei Wang, No.167 Beilishi Road, Xicheng District, Beijing 100037; 8610-88322539 (Shibua Zhao), 8610-88396773 (Wei Wang) (emails: zhaoshibua0202@126.com; drweiwang0728@hotmail.com). population [Siu]. It is associated with a high risk of proximal aorta dilation, which involves proximal arch (PArc) in $73 \%$ of BAV patients [Fazel]. PArc dilation bears an increased risk of life-threatening events, such as aortic dissection or rupture. Hemiarch replacement was recommended as a routine procedure in these patients [Fazel]. However, the complexity of such a procedure is associated with increased risks, such as prolonged operation and cerebral ischemic stroke [Etz]. The factors that contribute to the development of PArc dilation may influence surgical decisions. If these factors can be addressed, the PArc dilation may not require prosthetic replacement.

However, the possible contributing factors have not been systematically investigated. Fazel et al [Fazel] found that PArc dilation rarely occurs alone. Instead, it is usually concomitant with ascending aorta (AAo) dilation. Based on

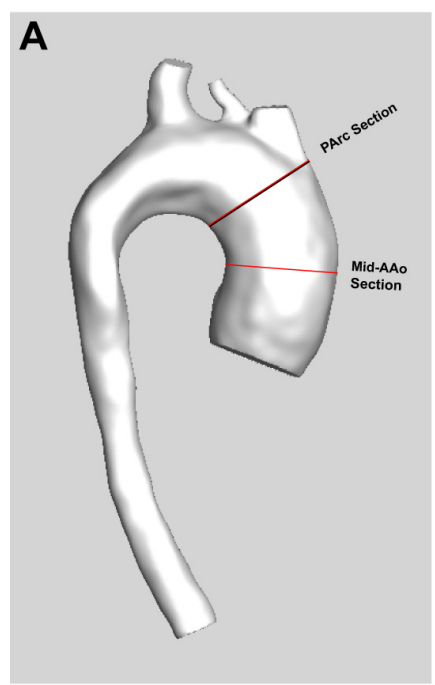

Oblique Sagittal

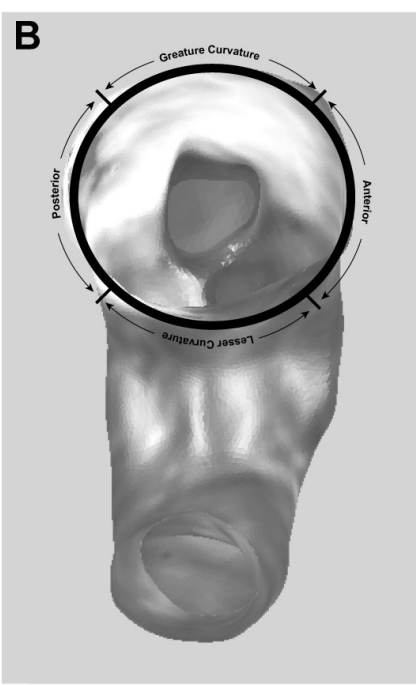

Axial
Figure 1. Schematic presentation of the measured levels at the proximal aorta and the definition of aortic regions in the circumferential direction in the aortic contour image derived from 4D-Flow MRI. A, The measured levels of aortic diameter were showed with the solid red line and the measured level of WSS were presented with the solid black line. B, The presentation of the definition of aortic regions in the circumferential direction. PArc: proximal arch; Mid-AAo: middle of ascending aorta. 


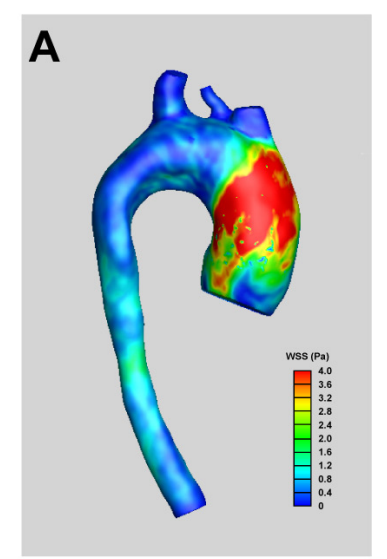

PArc Dilation
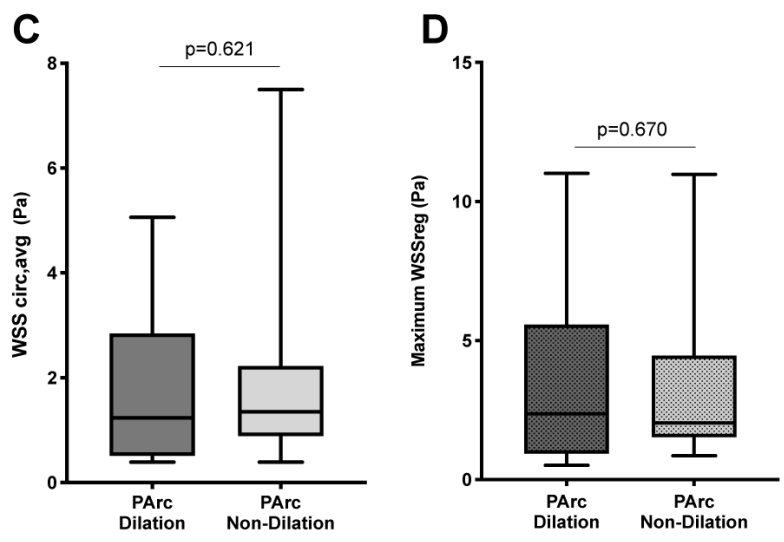

Figure 2. The comparison of WSS in PArc. A, B, Example of 4D-Flow $\mathrm{MRI}$ imaging with three-dimensional velocity encoding-derived aortic WSS map in BAV patients with or without PArc dilation. C, The comparison of WSScirc,avg in PArc section between the patients with and without PArc dilation. D, The comparison of maximum WSSreg between the two groups. WSS: wall shear stress; PArc: proximal arch; WSScirc,avg: contour-averaged circumferential wall shear stress; WSSreg: regional wall shear stress; $\mathrm{Pa}=\mathrm{N} / \mathrm{m}^{2}$.

this observation, they inferred that PArc dilation might be secondary to the upward extension of AAo dilation [Fazel]. Whereas, in BAV patients, Dux-Santoy et al observed that dilated PArc, compared with non-dilated PArc, had higher inplane rotational flow [Dux-Santoy]. They explained that the development of PArc dilation might be a result of the impact of abnormal valve-related hemodynamics. Although the two possible mechanisms have been speculated, there currently is no further evidence to evaluate them.

In this study, we evaluated the two possible contributing factors of PArc dilation using four-dimensional flow magnetic resonance imaging (4D-Flow MRI). Increased wall shear stress (WSS) that derived from abnormal blood flow could directly impact the aortic wall and result in medial elastin degeneration [Guzzardi]. Thus, the regional WSS and aortic diameters were measured in BAV patients with or without PArc dilation. We then compared the regional WSS between

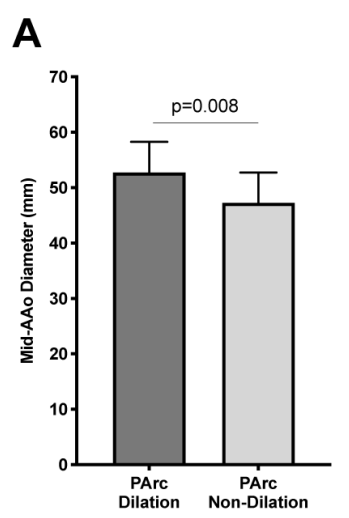

B
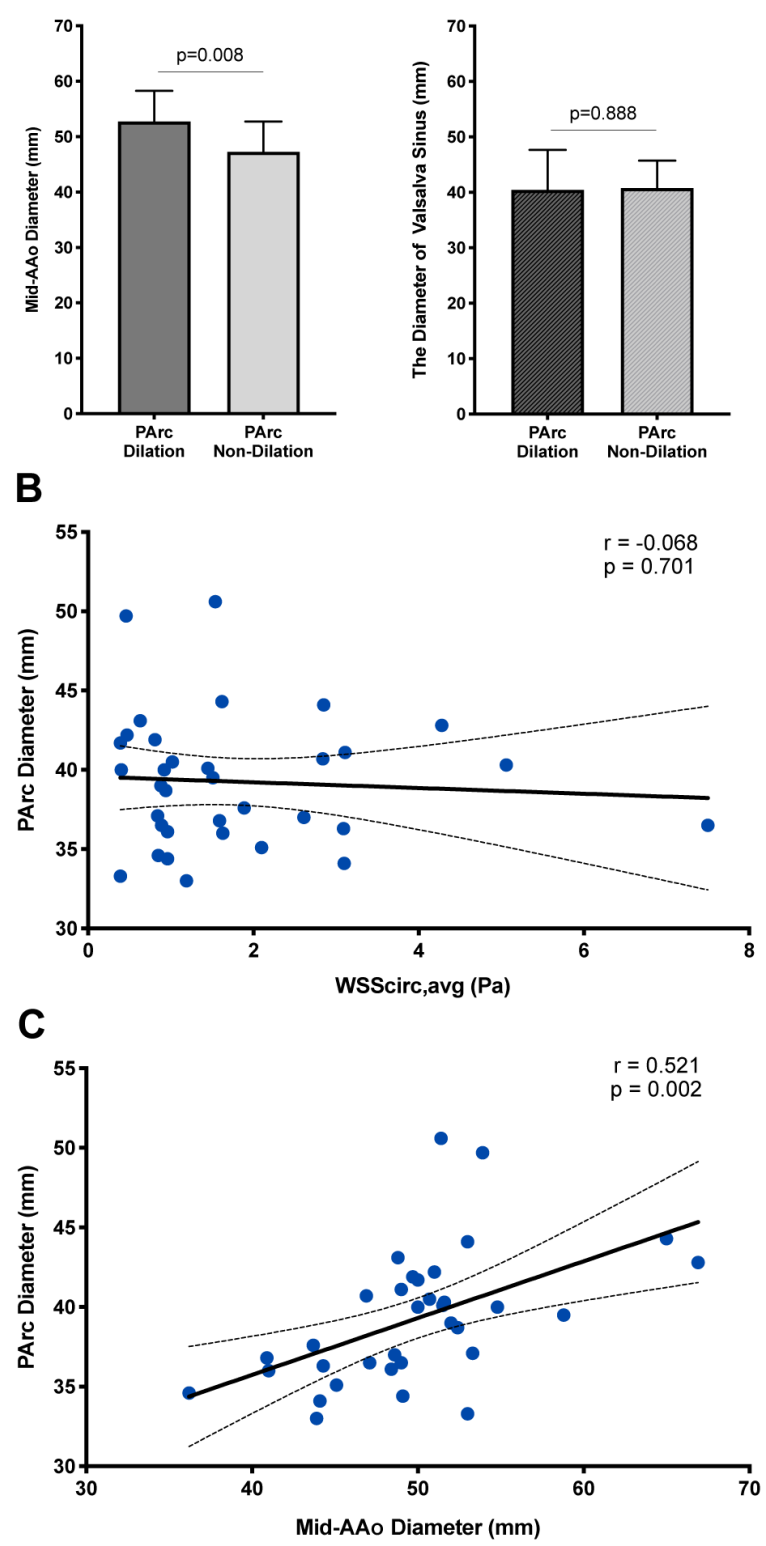

Figure 3. A, The comparison of the diameter of mid-AAo and Valsalva sinus between the patients with and without PArc dilation. B, The correlation analysis between WSScirc,avg and aortic diameter at PArc section. C, Mid-AAo diameter correlated with PArc diameter. Mid-AAo: middle of ascending aorta; PArc: proximal arch. WSScirc,avg: contouraveraged circumferential wall shear stress. Correlation coefficients $\mathrm{R}$, $\mathrm{P}$ values, lines of best fit (solid line), and lines of $95 \% \mathrm{Cl}$ (dashed line) are provided.

the two groups and analyzed the correlation between WSS and PArc diameter, as well as the correlation between PArc diameter and the diameter of middle AAo (mid-AAo). Our study aimed to investigate whether the two factors contribute to the development of PArc dilation. 
Table 1. Baseline characteristics

\begin{tabular}{lccc}
\hline Characteristics & PArc Dilation & PArc Non-Dilation & $P$ \\
\hline Men, $\mathrm{n}(\%)$ & $11(68.8)$ & $16(88.9)$ & .214 \\
Age, $y$ & $57.88 \pm 7.75$ & $50.72 \pm 14.41$ & .079 \\
Body mass index $\left(\mathrm{kg} / \mathrm{m}^{2}\right)$ & $24.93 \pm 3.31$ & $25.00 \pm 4.53$ & .962 \\
Body surface area $\left(\mathrm{m}^{2}\right)$ & $1.77 \pm 0.16$ & $1.81 \pm 0.24$ & .551 \\
Hypertension, $\mathrm{n}(\%)$ & $8(50.0)$ & $10(55.6)$ & 1.000 \\
Patterns of valvular & & & 1.000 \\
dysfunction, $\mathrm{n}(\%)$ & $12(75.0)$ & $13(72.2)$ & \\
AS & $4(25.0)$ & $5(27.8)$ & \\
AR & $46.50 \pm 30.67$ & $32.94 \pm 24.11$ & .159 \\
AV-mean gradient $(\mathrm{mmHg})$ & $59.81 \pm 7.76$ & $59.39 \pm 9.59$ & .695 \\
LVEF, \% & & & \\
\hline
\end{tabular}

Data are reported as mean \pm standard deviation or number (percentage). PArc: proximal arch; AS: aortic stenosis; AR: aortic regurgitation; AV: aortic valve; LVEF: left ventricular ejection fraction

\section{MATERIALS AND METHODS}

Study population: All enrolled patients signed an informed consent that was approved and granted by the ethics committee of Fuwai Hospital before enrollment. Between May 2017 and January 2019, 34 BAV patients with the dilation of the proximal aorta were enrolled and divided into two groups: PArc dilation $(\mathrm{N}=16)$ and PArc non-dilation $(\mathrm{N}=18)$. The patients with previous cardiac surgeries, a family history of aortopathy, suspected connective tissue diseases, and abdominal aortic aneurysm were excluded. 4D-Flow MRI was used to assess hemodynamic characteristics in the thoracic aorta.

4D-Flow MRI image acquisition: MRI examination was performed for all patients at a 3.0 Tesla MR scanner (Discovery MR750, GE Healthcare, Waukesha, Wis.) before the surgery. Aortic blood flow was assessed by 4D-Flow MRI with three-directional velocity encoding [Markl]. All data was obtained during free breathing using retrospective electrocardiograph gating in a sagittal-oblique volume covering the entire left ventricular outflow tract and thoracic aorta. Four NEX was used for suppressing respiratory artifacts. The scan parameters were as follows: TE: $2.2 \mathrm{~ms}$, TR: $4.4 \mathrm{~ms}$, bandwidth: $62.5 \mathrm{~Hz} /$ pixel, flip angle: $14^{\circ}$, temporal resolution: $35.2 \mathrm{~ms}$, field of view: $360 \times 252$ mm, matrix: $180 \times 126$, slice thickness: $4.8 \mathrm{~mm}$, reconstructed spatial resolution: $2.0 \times 2.0 \times 2.4 \mathrm{~mm}^{3}$, number of slices: $36 \pm 4$. Twenty-five reconstructed phases during one cardiac cycle; velocity encoding: 150-300 cm/s (based on two-dimensional phase-contrast MRI scout measurements).

WSS calculation: Image data of 4D-Flow MRI was postprocessed using the CardioSim software (Shengshi Technology, Co., Ltd, Hangzhou, China). Neighborhood variance method and neighborhood sign determination method were used for denoising 4D-Flow MRI velocity field [Huang]. In this way, the majority of noise can be removed to reveal the
Table 2. Regional WSS and aortic diameters

\begin{tabular}{lccc}
\hline & PArc Dilation & PArc Non-Dilation & $P$ \\
\hline WSS at PArc section (Pa) & & & \\
WSScirc,avg & $1.24[0.51-2.85]$ & $1.35[0.89-2.23]$ & 0.621 \\
Maximum WSSreg & $2.37[0.93-5.58]$ & $2.04[1.53-4.47]$ & 0.670 \\
WSSreg-GC & $0.93[0.54-2.48]$ & $1.15[0.39-1.64]$ & 0.798 \\
WSSreg-A & $1.73[0.57-3.22]$ & $1.61[0.68-2.78]$ & 1.000 \\
WSSreg-LC & $1.06[0.54-1.84]$ & $1.50[0.82-2.37]$ & 0.224 \\
WSSreg-P & $0.61[0.24-2.24]$ & $0.87[0.31-1.70]$ & 0.721 \\
Aortic diameters (mm) & & & \\
PArc & $42.69 \pm 3.23$ & $36.20 \pm 1.86$ & \\
Mid-Ascending Aorta & $52.76 \pm 5.51$ & $47.27 \pm 5.48$ & 0.008 \\
Valsalva Sinus & $40.48 \pm 7.17$ & $40.78 \pm 4.92$ & 0.888 \\
\hline
\end{tabular}

Continuous variables with a normal distribution are presented as the mean \pm standard deviation or median [IQR] when nonnormally distributed. P values showed comparison analyses between the groups, PArc dilation and PArc non-dilation. PArc: proximal arch; WSS: wall shear stress; WSScirc,avg: contour-averaged circumferential WSS; WSSreg: regional WSS; GC: greater curvature; A: anterior; LC: lesser curvature; P: posterior

profile of aorta. At last, a 3D aortic model was extracted and reconstructed based on the velocity field attached in anatomical images [Otsu; Kazhdan].

Generally, 4D-Flow MRI flow field contains a high level of noise, errors, and coarse resolution near the vessel's wall. Therefore, post-processing on the velocity field is necessary. In this paper, an advanced technique of experimental fluid mechanics, the divergence-free smoothing (DFS) method [Wang], was applied to the velocity field to reduce noise and fix errors. However, the available DFS methods only provide the methodology on applications of the external flow field [Wang], while the issue of using DFS in wall-bounded flows as blood flow in the aorta will be investigated in the current work. In a wall-bounded flow, the no-slip condition on the wall needs to be satisfied with DFS. Therefore, special treatment of discrete grids near the curved wall is required, and the most important thing is to establish the difference equation of divergence-free in the near wall region. For this reason, the Taylor expansion of the velocity at the nearest grid point from 4D-Flow MRI was adopted along the wall-normal direction to achieve the difference equation for near wall region. The aim of the DFS process is that the difference between optimized velocity field and original velocity field should be as small as possible. So, the objective function of the optimization process is established, which is subjected to the divergence of smoothed velocity field in the DFS method that needs to be zero [Wang; Garcia]. By addressing this optimization issue, smoothed velocity field can be obtained.

After the improvement of blood velocity field with modified DFS and aorta reconstruction, both the three- 
dimensional velocity field and the vessel wall are available for further calculation of WSS. To estimate the WSS, local coordinates of the curved wall surface were established. A fitting of binomial was then applied to the velocity field along the local wall-normal direction based on the Musker model [Kendall]. The formula of velocity profile near the wall was based on the vortex-bonding model [Kendall], which was determined with local flow properties of the wall friction velocity, blood density, and dynamic viscosity coefficient of the blood. Finally, the WSS was calculated following the formula, $W S S=\left.\mu \frac{d u}{d x}\right|_{y=0}$. Here, $\mu$ is the coefficient of viscosity and all coordinates and velocity components are local.

Definitions and measurements: PArc level was defined as the transection proximal to the innominate artery. The contour-averaged circumferential WSS (WSScirc,avg) was acquired at PArc level (Figure 1A). The sections at proximal aorta were equally divided into four parts in the circumferential direction: greater curvature and lesser curvature walls, as well as the anterior and posterior walls (Figure 1B). The regional WSS (WSSreg) of all parts was computed. The diameters of mid-AAo and PArc were measured using the images derived from 4D-Flow MRI (Figure 1A). The diameter at the level of the Valsalva sinus was obtained from axial half-Fourier single-shot turbo spin-echo scan sequence imaging of the thorax. PArc dilation was defined as the diameter of PArc $\geq 40 \mathrm{~mm}$ [Jackson]. Aortic valve dysfunction was evaluated using two-dimensional cardiac ultrasound, and the pattern of valve dysfunction was determined according to the guideline of valvular heart disease [Nishimura 2014].

Statistical analysis: Continuous variables were tested by Shapiro-Wilk test to evaluate whether the data is normally distributed. The data with a normal distribution was presented as mean \pm standard deviation, while those with a non-normal distribution were presented as median (interquartile range). Categorical variables are presented as number (n) and percentage (\%). Student's t-test and Mann-Whitney U test were used to compare the values between two groups. Chi-square test or Fisher's exact test was used to compare the frequencies between the groups. The correlation was analyzed by Pearson or Spearman correlation analysis. Statistical analyses were performed with SPSS version 25 (IBM Inc., New York, NY), and $P<.05$ was considered statistically significant (two-sided).

\section{RESULTS}

Baseline characteristics: The baseline characteristics are shown in Table 1. (Table 1) The mean age of all enrolled patients was $54.09 \pm 12.14$ years, and $79.4 \%(\mathrm{~N}=27)$ of these patients were males. There is no statistical difference in age and gender between the patients with and without PArc dilation ( $P=.079, P=.214$; respectively). No significant difference was observed in the patterns of aortic valve dysfunction between the two groups. Similarly, other demographics also did not show significant difference between the two groups.

No difference in WSS between patients with and without PArc dilation: The results of regional WSS are summarized in Table 2. Examples of aortic WSS map derived from 4D-Flow
MRI are shown in Figure 2A and Figure 2B. No significant difference was found in the WSScirc,avg on the PArc sections between the patients with and without PArc dilation $(P=.621)$ (Figure 2C), as well as no difference in the WSSreg (greater curvature, anterior, lesser curvature, and posterior) between the two groups $(P=.798, P=1.000, P=.224, P=.721$, respectively) (Table 2 ). There were no significant differences in the regional distribution of maximum WSSreg at circumferential direction between the two groups $(\mathrm{P}=.807)$. Furthermore, the maximum WSSreg were selected from the four regions, and no significant differences were observed in the maximum WSSreg between the patients with and without PArc dilation $(\mathrm{P}=.670)$ (Figure 2D).

PArc diameter correlated with mid-AAo diameter, but not regional WSS: Next, we investigated whether PArc dilation associated with AAo dilation. The results of the aortic diameters are presented in Table 2. The diameter of mid-AAo in patients with PArc dilation is higher than the patients without PArc dilation $(P=.008)$ (Figure $3 \mathrm{~A})$. There was no significant difference in the diameter of Valsalva sinus $(P=.888$, Figure $3 \mathrm{~A})$ between the two groups. The correlation was found between the diameters of PArc section and of mid-AAo section $(\mathrm{R}=0.521, P=.002$ (Figure 3C). However, the correlation analysis between PArc diameter and the WSScirc,avg showed that there was no significant difference $(\mathrm{R}=-0.068$, $P=.701)$ (Figure 3B).

\section{DISCUSSION}

In this study, aortic hemodynamics was visualized by means of 4D-Flow MRI. Few reports described the valve-related blood flow patterns (i.e. flow jet, helical flow, and vortices), or measured WSS in proximal aorta. It has been shown that WSS could directly impact on aortic wall leading to medial elastin degeneration [Guzzardi]. Dux-Santoy and his co-workers [Dux-Santoy] observed increased rotational flow located in the position of PArc, speculating that the dilation of PArc may be attributed to the blood flow turbulence. Interestingly, they also showed that BAV patients who suffered PArc dilation had lower regional axial WSS and increased regional circumferential WSS at the PArc comparing with the non-dilated patients [Dux-Santoy]. Farag et al. observed that aortic valve replacement did not alter the distribution and intensity of WSS on PArc [Farag; Farag]. These different findings implied that the hemodynamic factor should be re-evaluated with regard to its possible contribution to the development of PArc dilation. Besides, Park and his colleagues [Park] provided clinical evidence based on long-term follow-up for patients undergoing repair or replacement of the AAo, which showed that progressive PArc dilation was uncommon after AAo surgery.

Together, this challenged the speculation that valverelated hemodynamics contributed to the development of PArc dilation.

Our study compared the regional WSS between the two groups and analyzed the correlation between WSS and PArc diameter. No significant difference was found in the regional WSS between the patients with or without PArc dilation. We did 
not observe significant correlation between the WSScirc,avg and the diameter of its corresponding level, too. These results may suggest that WSS is not a contributing factor in the development of PArc dilation in BAV patients. Furthermore, we compared the mid-AAo diameter of the two groups and analyzed the association between the diameter of mid-AAo and PArc. We found that the diameter of mid-AAo in patients with PArc dilation significantly is higher than that without the PArc dilation. Besides, the diameter of PArc significantly correlated with the diameter of mid-AAo. These results indicated that PArc dilation might be secondary to the upward extension of AAo dilation.

Embryologic evidence also supports our findings. Based on the Edwards' hypothetical double arch diagram and the Rathke's diagram of aortic arches [Edwards; Edwards; Stojanovska], the branchial apparatus consists of six branchial arches; the fourth one becomes the aortic arch [Murillo]. However, the AAo originate from the truncus arteriosus and primordial aortic sac [Stojanovska]. It is clear that AAo and aortic arch originated from different portions of the embryo, which could explain the disease-related heterogeneity between the two segments of the aorta. The region of PArc is a transition region from AAo to aortic arch, which could be influenced by the dilation of proximal tubular ascending aorta. These information are consistent with the clinical observation that AAo dilation rarely involved the aorta distal to the innominate artery [Fazel].

The partial arch replacement was recommended together with AAo repair in ascending aortic aneurysms involving the PArc, using right subclavian/axillary artery inflow and hypothermic circulatory arrest [Hiratzka 2010]. With the improvement of surgical techniques, the operative morbidity and mortality are low in patients who underwent aortic arch surgery, albeit higher than those of AAo replacement alone [Etz]. In our study, the results revealed that WSS is not a contributing factor in proximal arch dilation in BAV patients. The enlargement of PArc might be secondary to the upward extension of the AAo dilation. The contributing factor of the development of PArc dilation could be removed from the surgery of prosthetic replacement of AAo alone, hence, a less aggressive strategy to hemiarch replacement might be appropriate.

Limitations: Our study did not find the correlation between WSS and PArc diameter. The relatively small study population size will increase the probability of making a Type II error, which may fail to reject the null hypothesis of no association. Further studies with larger sample sizes are needed to confirm these results. Besides, only BAV patients were included in this study to evaluate whether WSS is a contributing factor in the development of PArc dilation. An additional control group of patients with normal tricuspid aortic valve may be beneficial to contextualize our findings.

\section{CONCLUSIONS}

Our study shows WSS is not a contributing factor in the development of PArc dilation. We observed that the PArc diameter correlated with mid-AAo diameter, which indicated that the enlargement of PArc may be a result of the upward extension of AAo dilation. AAo replacement could remove the contributing factor of PArc dilation, hence, a selective approach to transverse PArc replacement might be appropriate.

\section{REFERENCES}

Dux-Santoy L, Guala A, Teixido-Tura G, et al. 2019. Increased Rotational Flow in the Proximal Aortic Arch Is Associated With Its Dilation in Bicuspid Aortic Valve Disease. Eur Heart J Cardiovasc Imaging. 20(12):1407-1417.

Edwards JE. 1948. Anomalies of the Derivatives of the Aortic Arch System. Med Clin North Am. 32:925-949.

Edwards JE. 1953. Malformations of the Aortic Arch System Manifested as Vascular Rings. Lab Invest. 2(1):56-75.

Etz CD, Plestis KA, Homann TM, et al. 2008. Reoperative Aortic Root and Transverse Arch Procedures: A Comparison with Contemporaneous Primary Operations. J Thorac Cardiovasc Surg. 136 (4):860-867, 867.e1-3.

Farag ES, Schade EL, van Ooij P, et al. 2019. Bileaflet Mechanical Aortic Valves Do Not Alter Ascending Aortic Wall Shear Stress. Int J Cardiovasc Imaging. 35(4):703-710.

Farag ES, Vendrik J, van Ooij P, et al. 2019. Transcatheter Aortic Valve Replacement Alters Ascending Aortic Blood Flow and Wall Shear Stress Patterns: A 4D Flow MRI Comparison With Age-Matched Elderly Controls. Eur Radiol. 29(3):1444-1451.

Fazel SS, Mallidi HR, Lee RS, et al. 2008. The Aortopathy of Bicuspid Aortic Valve Disease Has Distinctive Patterns and Usually Involves the Transverse Aortic Arch. J Thorac Cardiovasc Surg. 135(4):901-907, 907. e1-2.

Garcia D. 2010. Robust Robust Smoothing of Gridded Data in One and Higher Dimensions With Missing Values. Comput Stat Data Anal. 54(4):1167-1178.

Guzzardi DG, Barker AJ, van Ooij P, et al. 2015. Valve-Related Hemodynamics Mediate Human Bicuspid Aortopathy: Insights From Wall Shear Stress Mapping. J Am Coll Cardiol. 66(8):892-900.

Hiratzka LF, Bakris GL, Beckman JA, et al. 2010. ACCF/AHA/AATS/ ACR/ASA/SCA/SCAI/SIR/STS/SVM Guidelines for the Diagnosis and Management of Patients With Thoracic Aortic Disease: A Report of the American College of Cardiology Foundation/American Heart Association Task Force on Practice Guidelines American Association for Thoracic Surgery American College of Radiology American Stroke Association Society of Cardiovascular Anesthesiologists Society for Cardiovascular Angiography and Interventions Society of Interventional Radiology Society of Thoracic Surgeons and Society for Vascular Medicine. Circulation. 121(13):e266-369.

Huang TS, Yang GJ, Tang GY. 1979. A Fast Two-dimensional Median Filtering Algorithm. IEEE Trans on Acoustic Speech \& Signal Processing 27(1):13-18.

Jackson V, Petrini J, Caidahl K, et al. 2011. Bicuspid Aortic Valve Leaflet Morphology in Relation to Aortic Root Morphology: A Study of 300 Patients Undergoing Open-Heart Surgery. Eur J Cardiothorac Surg. 40(3):e118-124.

Kazhdan M, Hoppe H. 2013. Screened Poisson Surface Reconstruction. ACM Trans. Graph 32(3): 29(1-13).

Kendall A, Koochesfahani M. 2008. A Method for Estimating Wall Friction in Turbulent Wall-Bounded Flows. Exp Fluids 44:773-780. 
Markl M, Harloff A, Bley TA, et al. 2007. Time-resolved 3D MR Velocity Mapping at 3T: Improved Navigator-Gated Assessment of Vascular Anatomy and Blood Flow. J Magn Reson Imaging. 25(4):824-831.

Murillo H, Lane MJ, Punn R, et al. 2012. Imaging of the Aorta: Embryology and Anatomy. Semin Ultrasound CT MR. 33(3):169-190.

Nishimura RA, Otto CM, Bonow RO, et al. 2014. AHA/ACC Guideline for the Management of Patients With Valvular Heart Disease: Executive Summary: A Report of the American College of Cardiology/American Heart Association Task Force on Practice Guidelines. J Am Coll Cardiol. 63(22):2438-2488.

Otsu N. 1979. A Threshold Selection Method from Gray-Level
Histograms. IEEE Trans Syst Man Cybern 9(1): 62-66.

Park CB, Greason KL, Suri RM, et al. 2011. Should the Proximal Arch Be Routinely Replaced in Patients With Bicuspid Aortic Valve Disease and Ascending Aortic Aneurysm? J Thorac Cardiovasc Surg. 142(3):602-607.

Siu SC, Silversides CK. 2010. Bicuspid Aortic Valve Disease. J Am Coll Cardiol. 55(25):2789-2800.

Stojanovska J, Cascade PN, Chong S, et al. 2012. Embryology and Imaging Review of Aortic Arch Anomalies. J Thorac Imaging. 27(2):73-84.

Wang CY, Gao Q, Wang HP, et al. 2016. Divergence-Free Smoothing for Volumetric PIV Data. Exp Fluids. 57: 15(1-23). 